\title{
An investigation of flow behaviour at the junction of rectangular channels
}

by

\author{
N. B. Webber, B.Sc.(Eng.), A.M.I.C.E., M.A.S.C.E., A.M.I.W.E., \\ A.M.I.Struct.E.
}

and

C. A. Greated, B.Sc.(Eng.), Ph.D.

Dr M. B. Abbott (Reader for Hydraulics, International Courses, Delft Technological University) had followed the Authors' work on flow pattern computations with interest. Their demonstration that a potential flow and Helmholtz free streamline theory could, under certain circumstances, provide acceptable flow patterns and optimum profiles already in itself extended the scope of the designer. It would no doubt lead to other applications. However, such applications would have to proceed with considerable caution, owing to the inherent assumptions, mentioned in $\S 27$ and \$ 31. Since direct model verification was not always convenient, could the Authors suggest some criteria, preferably suited to the numerical scheme, for estimating the degree of approximation under given circumstances? Dr Abbott had in mind something like the third derivative checks on vertical accelerations used in long wave computations.

35. The methods of Thom and Apelt, like so many numerical schemes for elliptic equations, could be painfully slow. In fact, unless one had unrestricted computer time, some means of accelerating the convergence of these methods seemed to be essential. So far as Dr Abbott was aware, the Authors' work constituted the first application of the powerful convergence acceleration techniques of Carré and Kulsrud, whereby computer time could be cut to only a few percent of that otherwise required. ${ }^{9,10}$ He was surprised to see no mention of these methods in the present Paper. Would the Authors really propose such computations, especially on a repetitive basis, without convergence acceleration?

36. For design purposes, many different possibilities had to be investigated, thus putting a premium on very general machine programs. Could the Authors' program be generalized to cover a range of intersection angles and different channel widths?

37. In $\$ 31$ mention was made of supercritical flow conditions. It should be mentioned that in this case the flow equations were principally hyperbolic, so that the method described by the Authors could not then apply. Comparable supercritical flow solutions could doubtless be constructed, using the method of characteristics with free streamlines, but the method involved would then be quite different from that of the Authors.

The Authors were grateful to Dr Abbott for his constructive comments. Convergence acceleration techniques had been used in all the iterative calculations and it had been found that a significant reduction in computing time resulted. This had been especially true for the calculation of separated flow through the junction, where it had been necessary to use about 1500 mesh points in order to obtain an accurate solution. The rate of convergence of the Laplace iterative process decreased rapidly with increase in the number of mesh points and it was even slower if derivative conditions were used at the boundaries. It was in these circumstances, then, that the acceleration technique was particularly useful. 
39. The method of convergence acceleration was based on the over-relaxation process first devised by Thom and Southwell, but the problem was to calculate the value of the optimum over-relaxation or accelerating factor $W_{\text {opt }}$ for a given set of data. In general, this could only be done when the results of a number of iterations were known. Thus the procedure was to perform a few iterations with some convenient value of $W$ and then to calculate an approximate value of $W_{\mathrm{opt}}$ from the results. This value, or one related to it, was used for the next set of iterations, after which a better approximation to $W_{\text {opt }}$ was found, and so on successively.

40. The papers quoted by Dr Abbott ${ }^{8,10}$ described methods of calculating values. The method of Kulsrud was the simplest, but Carre's method had been found to be more reliable, and had therefore been employed in the present computations. Both methods were based on the same criterion. If a norm was used for calculating the rate of convergence (usually taken as the first power norm, which was the sum of the numerical values of the displacement vector elements), the ratio of the norms on two consecutive iterations converged towards the dominant eigenvalue $\lambda_{1}$ of the successive over-relaxation matrix. If the iterations are performed with an accelerating factor $W$, the optimum value $W_{\text {opt }}$ was given by

$$
W_{\mathrm{opt}}=\frac{2}{1+\left[1-\left(\lambda_{1}+W-1\right)^{2} /\left(\lambda_{1} W^{2}\right)\right]^{1 / 2}} \quad . \quad . \quad .
$$

41. Difficulties in the calculation of $W_{\text {opt }}$ arose mainly from the fact that when $W$ was chosen greater than $W_{\text {opt }}$ all the eigenvalues became complex. Indeed, instead of $W$ converging most rapidly towards the correct value of $W_{\text {opt }}$ when $W$ was chosen close to $W_{\text {opt }}$, it was found that the most favourable procedure was to utilize the value of $W$ corresponding to the maximum value of the dominance ratio $\left|\lambda_{2}\right| \div\left|\lambda_{1}\right|$, where $\lambda_{2}$ was the second largest eigenvalue. This method of analysis was incorporated in a computer program for solving the flow patterns at junctions having different intersection angles.

42. The computations were based on the assumption of a two-dimensional model and therefore were only applicable to low Froude numbers. A complete analysis of the errors arising would have been beyond the scope of the Paper, but it could be seen from Fig. 5 that the change in depth across the junction was small even at quite high Froude numbers.

43. A number of tests had been made involving the supercritical flow condition and it was hoped that a paper would be published describing a simplified analysis.

\section{REFERENCES}

9. CARRE B. A. The determination of the optimum accelerating factor for successive over-relaxation. Computer J., 1961, 4, 73-78.

10. KulsRud H. E. A practical technique for the determination of the optimum relaxation factor of the successive over-relaxation method. Comm. Assoc. Comp. Mach., 1961, 4, 184-187. 